\title{
The Insulin-like Growth Factor-1 (G>A) and 5,10-methylenetetrahydrofolate Reductase (C677T) Gene Variants and the Serum Levels of Insulin-like Growth Factor-1, Insulin, and Homeostasis Model Assessment in Patients with Acne Vulgaris
}

Sakineh Zinati-Saeed ${ }^{1}$, Ebrahim Shakiba ${ }^{1}$, Ziba Rahimi ${ }^{2}$, Mona Akbari ${ }^{2}$, Fariba Najafi ${ }^{3}$, Fariborz Bahrehmand ${ }^{2}$, Asad Vaisi-Raygani ${ }^{4}$, Zohreh Rahimi ${ }^{2 *}$ iD, Ali Ebrahimi ${ }^{3}$, Mehrali Rahimi ${ }^{5}$

1. Department of Clinical Biochemistry, Medical School, Kermanshah University of Medical Sciences, Kermanshah, Iran 2. Medical Biology Research Center, Research Institute for Health Technology, Kermanshah University of Medical Sciences, Kermanshah, Iran 3. Department of Dermatology, Medical School, Kermanshah University of Medical Sciences, Kermanshah, Iran

4. Fertility and Infertility Research Center, Medical School, Kermanshah University of Medical Sciences, Kermanshah, Iran

5. Department of Internal Medicine, Medical School, Kermanshah University of Medical Sciences, Kermanshah, Iran

\section{KEYWORDS \\ Acne vulgaris, \\ Methylenetetrahydrofolate reductase, \\ Insulin-like growth factor-1, insulin, \\ Homeostasis model assessment}

Scan to discover online

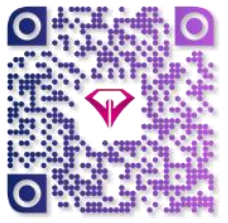

\begin{tabular}{c}
\hline Main Subjects: \\
Clinical Chemistry \\
\hline Received 7 Apr 2019; \\
Accepted 22 Sep 2019; \\
Published Online 26 Dec 2019; \\
\hline
\end{tabular}

10.30699/IJP.2019.105695.2098

PMCID: PMC6995677

PMID: $\quad 32095146$

\section{ABSTRACT}

Background \& Objective: To find an association between gene variants of insulinlike growth factor-1 (IGF-1) and 5,10-methylenetetrahydrofolate reductase (MTHFR) with the risk of acne vulgaris (AV).

Methods: In a case-control study, we investigated $150 \mathrm{AV}$ patients and 148 healthy individuals (aged 18-25 years) for the IGF-1 G>A and MTHFR C677T polymorphisms, as well as the serum levels of IGF-1, insulin, and the homeostasis model assessment of insulin resistance (HOMA-IR). The serum biochemical parameters and the genotypes of IGF-1 G>A and MTHFR C677T were detected by using appropriate kits and polymerase chain reaction-restriction fragment length polymorphism (PCR-RFLP) methods, respectively.

Results: The frequencies of IGF-1 and the MTHFR polymorphisms were not significantly different comparing patients and controls. The serum level of IGF-1 was $179.8 \pm 72.8 \mu \mathrm{g} / \mathrm{L}$ in AV patients compared to $164.6 \pm 63.7 \mu \mathrm{g} / \mathrm{L}$ in controls $(P=0.056)$. The serum level of insulin in female patients was significantly higher than controls. The HOMA was $3.54 \pm 5.6$ in patients compared to $1.16 \pm 1.4(P<0.001)$ in controls. Significantly higher levels of fasting blood sugar (FBS), total cholesterol, and lowdensity lipoprotein-cholesterol (LDL-C) were detected in female patients than controls. However, the level of estradiol was significantly lower in female patients than in controls. In females, the presence of the MTHFR $\mathrm{T}$ allele was associated with significantly higher levels of FBS and LDL-C, as well as a significantly lower level of estradiol compared to those carriers of the $\mathrm{C}$ allele.

Conclusion: We found the absence of an association between IGF-1 and MTHFR polymorphisms with the risk of AV. However, increased insulin, IGF-1, and HOMA levels in AV patients indicated the effect of insulin and insulin resistance in the risk of AV and its severity.

Corresponding Information:

Zohreh Rahimi, PhD; Professor of Clinical Biochemistry, Medical Biology Research Center, Research Institute for Health Technology, Daneshgah Avenue, Kermanshah, Iran Email: zrahimi@kums.ac.ir

Copyright (C) 2020. This is an open-access article distributed under the terms of the Creative Commons Attribution- 4.0 International License which permit Share, copy and redistribution of the material in any medium or format or adapt, remix, transform, and build upon the material for any purpose, even commercially.

\section{Introduction}

Acne vulgaris (AV) is a chronic inflammatory skin disease, which is particularly common during adolescence and in young adults. This disease impairs various aspects of life quality (1). Clinical features of AV include seborrhea (excess grease), inflammation, abnormal follicular keratinization, and various degrees of scarring. The AV appears to be influenced by different factors, such as hereditary and genetics, nourishing, and environmental factors (2). Since AV reduces self-reliance in most teenage patients, its diagnosis and treatment are very important.

The insulin hormone has various roles in body metabolism. The insulin gene is located at the end of the short arm of chromosome 11 , synthesized in $\beta$ cells of the pancreas, and released in response to blood glucose to decrease it (3)

The gene of insulin-like growth factor-1 (IGF-1) is located on chromosome 12 and with similar structure 
to insulin. Insulin, growth hormone, and IGF-1 are effective factors in stimulating AV. However, IGF-1 is a stronger stimulant for sebum production and development of AV compared to insulin. The insulin and IGF-1 signaling is increased during puberty by a carbohydrate-rich diet, which may prompt the AV (4). The 5, 10-methylenetetrahydrofolate reductase (MTHFR) plays a key role in folate metabolism and synthesis of DNA. The C677T polymorphism of the MTHFR gene results in an alanine to valine alteration at position 222 of the polypeptide, which reduces the activity of the MTHFR (5). Severe MTHFR deficiency is associated with hyperhomocysteinemia, and the presence of MTHFR C677T polymorphism due to decreased MTHFR activity increases the susceptibility to the onset and progression of diabetic nephropathy in type 2 diabetes mellitus (6).

The present study aimed to investigate the possible association between variants of the MTHFR and IGF1 genes, as well as the levels of IGF-1, insulin, and homeostasis model assessment (HOMA) with the risk and severity of AV in a population from Kermanshah province in Western Iran.

\section{Materials and Methods Sample}

A total of 150 patients with mild-, moderate-, and severe-AV and 148 healthy individuals without symptoms and history of AV were studied in this research. Basic information of each individual, including the age, sex, weight, height, age of disease onset, period of disease, family history of $\mathrm{AV}$, severity of $\mathrm{AV}$ symptoms, and blood pressure were collected. All individuals were from Kermanshah province in Western Iran. AV grading area was defined according to the Global Acne Grading System, which divides the face, chest, and upper back into six areas, and assigns a number to each area based on the surface area, distribution, and density of pilosebaceous units (7).

The study was approved by the Ethics Committee of Kermanshah University of Medical Sciences and was in accordance with the principles of the Declaration of Helsinki II.

\section{Biochemical and Hormone Assay}

The blood sample was collected after overnight fasting. The levels of serum fasting blood sugar (FBS) triglyceride (TG), cholesterol, low-density lipoproteincholesterol (LDL-C), and high-density lipoproteincholesterol (HDL-C) were measured by using Bionic Diagnostic Kits (Iran) on Mindray BS-480 Chemistry Analyzer (China). Insulin and IGF-1 were evaluated by Monobind and Diametra hormone ELISA kits (made in USA and Italy respectively). The serum level of estradiol, in the mid-follicular phase of the menstrual cycle, was measured via the chemiluminescent method by using the Abbott Architect i1000 (Abbott Laboratory, USA).
Also, the homeostasis model assessment of insulin resistance (HOMA-IR) was calculated as follows:

$$
H O M A-I R=\frac{G l u \cos e(\mu U / m l) \times \operatorname{Insulin}(\mathrm{mg} / \mathrm{dl})}{405}
$$

\section{DNA Analysis}

Genomic DNA was extracted from peripheral leukocytes of ethylenediaminetetraacetic acid (EDTA)treated whole blood by the phenol-chloroform method (8). The G>A variant of the IGF-1 gene (rs6214) was amplified via polymerase chain reaction (PCR). The obtained PCR product with190-bp was digested by Hin 1 II (NlaIII) at $37^{\circ} \mathrm{C}$ for overnight; then, fragments were separated by $3 \%$ agarose gel (9).

The C677T polymorphism of the MTHFR gene (rs1801133) was amplified via PCR and subsequent digestion with Hinf I, as previously described (10).

\section{Statistical Analysis}

The statistical package for social sciences (SPSS) 16 (SPSS Inc., Chicago, IL, USA) was used for statistical analysis. The frequencies of alleles of both polymorphisms were calculated by the chromosome counting method. The $\chi^{2}$ test was used to calculate the significance of difference in the frequencies of genotypes and alleles of both polymorphisms between patient and control groups A two-tailed student's t-test and analysis of variance (ANOVA) were used to compare quantitative data. The P-value of less than $5 \%$ was considered as a statistically significant level.

\section{Results}

The biochemical characteristics of patients and controls are demonstrated in Table 1. As indicated in Table 1, both patient and control groups were agematched $(P=0.29)$. The serum level of insulin in AV patients $(16.7 \pm 26 \mu \mathrm{U} / \mathrm{mL})$ was significantly higher than in normal ones $(5.48 \pm 5.5 \mu \mathrm{U} / \mathrm{mL}, P<0.001)$. However, a near to significant difference, between IGF-1 serum levels of cases $(179.8 \pm 72 \mu \mathrm{g} / \mathrm{L})$ and controls $(164.6 \pm 63 \mu \mathrm{g} / \mathrm{L})$, was found $(P=0.056)$. The FBS level was $80.0 \pm 10.9 \mathrm{mg} / \mathrm{dL}$ in all AV patients compared to $77.5 \pm 17.0 \mathrm{mg} / \mathrm{dL}$ in controls $(P=0.94)$. HOMA-IR was significantly higher in AV patients (3.54 $\pm 5.6, P<0.001)$ than in controls $(1.16 \pm 1.4)$. The HDL-C serum level was significantly higher in patients $(48.5 \pm 12.9 \mathrm{mg} / \mathrm{dL})$ than in controls $(41.2 \pm 10.5 \mathrm{mg} / \mathrm{dL}$, $P<0.001)$. Parameters were compared in both sexes between the patients and the controls (Table 2). Insulin and HOMA-IR values were significantly higher in female patients than in female controls. Significantly higher levels of FBS, total cholesterol, and LDL-C were detected in female patients than in female controls. However, the level of estradiol was significantly lower in female patients $(88.4 \pm 81.9$ $\mathrm{pg} / \mathrm{mL})$ than in female controls $(118 \pm 86.9 \mathrm{pg} / \mathrm{mL}$, $P=0.004$ ) (Table 2). As indicated in Table 2, among males, only the cholesterol level was significantly higher in controls than in patients. 
Table 1. Characteristics of AV patients and controls

\begin{tabular}{|c|c|c|c|c|c|}
\hline Variables & Mild AV & Moderate AV & Severe AV & $\begin{array}{l}\text { All AV patients } \\
\quad(\mathrm{n}=150)\end{array}$ & Controls $(n=150)$ \\
\hline \multirow{2}{*}{ Age (years) } & $\mathrm{n}=53$ & $n=43$ & $\mathrm{n}=54$ & $22.2 \pm 4.6$ & \multirow{2}{*}{$22.7 \pm 4.0$} \\
\hline & $22.1 \pm 3.3$ & $23.7 \pm 5.4$ & $21.0 \pm 4.4$ & $P=0.29$ & \\
\hline \multirow{2}{*}{ BMI $\left(\mathrm{Kg} / \mathrm{m}^{2}\right)$} & $P=0.82$ & $P=0.55$ & $P=0.07$ & $23.4 \pm 6.6$ & \multirow{2}{*}{$22.4 \pm 3.6$} \\
\hline & $22.4 \pm 3.3$ & $24.6 \pm 10.4$ & $23.2 \pm 4.4$ & $P=0.13$ & \\
\hline \multirow{2}{*}{ Insulin $(\mu \mathrm{U} / \mathrm{ml})$} & $\mathrm{P}=1.0$ & $\mathrm{P}=0.08$ & $\mathrm{P}=0.77$ & $16.7 \pm 26$ & \multirow{2}{*}{$5.48 \pm 5.5$} \\
\hline & $16.7 \pm 23.4$ & $14.9 \pm 15.6$ & $18.5 \pm 34.4$ & $P<0.001$ & \\
\hline \multirow{2}{*}{ IGF-1 $(\mu \mathrm{g} / \mathrm{L})$} & $P=0.002$ & $P=0.018$ & $P<0.001$ & $179.8 \pm 72.8$ & \multirow{2}{*}{$164.6 \pm 63.7$} \\
\hline & $183 \pm 4.4$ & $179.7 \pm 70.4$ & $176.8 \pm 74.5$ & $P=0.056$ & \\
\hline \multirow{2}{*}{ HOMA } & $P=0.35$ & $P=0.56$ & $P=0.68$ & $3.54 \pm 5.6$ & \multirow{2}{*}{$1.16 \pm 1.4$} \\
\hline & $3.68 \pm 5.06$ & $3.0 \pm 3.2$ & $3.9 \pm 7.6$ & $P<0.001$ & \\
\hline \multirow{2}{*}{$\begin{array}{c}\text { Systolic blood } \\
\text { Pressure (mmHg) }\end{array}$} & $\mathrm{P}=0.002$ & $\mathrm{p}=0.057$ & $\mathrm{P}<0.001$ & $101.8 \pm 12.6$ & \multirow{2}{*}{$101.5 \pm 13.6$} \\
\hline & $99.1 \pm 11.9$ & $105.1 \pm 13.5$ & $101.3 \pm 12.1$ & $P=0.89$ & \\
\hline \multirow{2}{*}{$\begin{array}{l}\text { Diastolic blood } \\
\text { Pressure (mmHg) }\end{array}$} & $P=0.7$ & $P=0.4$ & $P=0.99$ & $71.3 \pm 9.5$ & \multirow{2}{*}{$73.7 \pm 12.3$} \\
\hline & $69.6 \pm 8.7$ & $74 \pm 9.5$ & $70.6 \pm 9.8$ & $\mathrm{P}=0.069$ & \\
\hline \multirow{2}{*}{ FBS (mg/dL) } & $\mathrm{P}=0.12$ & $\mathrm{P}=0.99$ & $P=0.29$ & $80.0 \pm 10.9$ & \multirow{2}{*}{$77.5 \pm 17.0$} \\
\hline & $78.7 \pm 8.9$ & $78.5 \pm 9.7$ & $82.5 \pm 13$ & $\mathrm{P}=0.12$ & \\
\hline \multirow{2}{*}{$\begin{array}{l}\text { Cholesterol } \\
(\mathrm{mg} / \mathrm{dL})\end{array}$} & $P=0.94$ & $P=0.97$ & $P=0.12$ & $130.2 \pm 30.4$ & \multirow{2}{*}{$126 \pm 30.3$} \\
\hline & $134.5 \pm 30.6$ & $128.6 \pm 27$ & $127.2 \pm 32.6$ & $P=0.24$ & \\
\hline \multirow{2}{*}{$\mathrm{TG}(\mathrm{mg} / \mathrm{dL})$} & $P=0.31$ & $P=0.96$ & $P=0.99$ & $81.9 \pm 51.4$ & \multirow{2}{*}{$83.9 \pm 42.4$} \\
\hline & $82.3 \pm 54.1$ & $75.1 \pm 35.6$ & $86.9 \pm 59.1$ & $P=0.71$ & \\
\hline \multirow{2}{*}{ HDL-C (mg/dL) } & $P=0.99$ & $P=0.71$ & $P=0.97$ & $48.5 \pm 12.9$ & \multirow{2}{*}{$41.2 \pm 10.5$} \\
\hline & $49.3 \pm 12.9$ & $48.7 \pm 14.6$ & $47.7 \pm 11.4$ & $P<0.001$ & \\
\hline \multirow{2}{*}{$\begin{array}{c}\text { LDL-C }(\mathrm{mg} / \mathrm{dL}) \\
\text { Variables }\end{array}$} & $P<0.001$ & $P=0.002$ & $P=0.004$ & $74.6 \pm 25$ & \multirow{2}{*}{$69.8 \pm 22.3$} \\
\hline & $78.0 \pm 26.7$ & $74.4 \pm 22.4$ & $71.6 \pm 25.5$ & $P=0.09$ & \\
\hline
\end{tabular}

AV: Acne vulgaris; BMI: Body mass index; IGF-1: Insulin-like growth factor-1; HOMA-IR: Homeostasis model assessment of insulin resistance; FBS: Fasting blood sugar; TG: Triglycerides; HDL-C: High-density lipoprotein-cholesterol; LDL-C: Low-density lipoprotein-cholesterol

Table 2. Characteristics of males and females in both groups of patients and controls

\begin{tabular}{|c|c|c|c|c|}
\hline Variables & Female Patients & Female Controls & Male Patients & \\
\hline \multirow{2}{*}{ Insulin $(\mu \mathrm{U} / \mathrm{ml})$} & $15.9 \pm 18.6$ & \multirow{2}{*}{$5.3 \pm 5.7$} & & $6.0 \pm 4.8$ \\
\hline & $P<0.001$ & & $22.4 \pm 52.6$ & $P=0.16$ \\
\hline \multirow{2}{*}{ IGF-1 $(\mu \mathrm{g} / \mathrm{L})$} & $179.9 \pm 75.3$ & \multirow{2}{*}{$165.5 \pm 68.6$} & $179.2 \pm 56.6$ & \multirow{2}{*}{$162 \pm 46.4$} \\
\hline & $P=0.12$ & & $P=0.24$ & \\
\hline \multirow{2}{*}{ HOMA } & $3.33 \pm 3.9$ & \multirow{2}{*}{$1.1 \pm 1.5$} & $4.9 \pm 11.5$ & \multirow{2}{*}{$1.3 \pm 1$} \\
\hline & $P<0.001$ & & $P=0.17$ & \\
\hline \multirow[b]{2}{*}{$\mathrm{FBS}(\mathrm{mg} / \mathrm{dL})$} & $80.3 \pm 10.3$ & \multirow{2}{*}{$76.4 \pm 17.7$} & $88.3 \pm 8.8$ & \multirow{2}{*}{$85.4 \pm 8.5$} \\
\hline & $P=0.027$ & & $P=0.14$ & \\
\hline \multirow{2}{*}{$\begin{array}{l}\text { Cholesterol } \\
(\mathrm{mg} / \mathrm{dL})\end{array}$} & $134.8 \pm 31.9$ & \multirow{2}{*}{$124.3 \pm 28.09$} & $125.9 \pm 24.6$ & \multirow{2}{*}{$140.2 \pm 31.6$} \\
\hline & $P=0.003$ & & $P=0.027$ & \\
\hline
\end{tabular}




\begin{tabular}{|c|c|c|c|c|}
\hline Variables & Female Patients & Female Controls & Male Patients & \\
\hline \multirow{2}{*}{$\mathrm{TG}(\mathrm{mg} / \mathrm{dL})$} & $82.1 \pm 50.4$ & \multirow{2}{*}{$79.6 \pm 38.8$} & $93.6 \pm 56.8$ & \multirow{2}{*}{$104 \pm 44.6$} \\
\hline & $P=0.62$ & & $P=0.39$ & \\
\hline \multirow{2}{*}{ HDL-C (mg/dL) } & $50.3 \pm 13.1$ & \multirow{2}{*}{$41.8 \pm 9.8$} & $44.7 \pm 9.2$ & \multirow{2}{*}{$42 \pm 12$} \\
\hline & $P<0.001$ & & $P=0.26$ & \\
\hline \multirow[b]{2}{*}{ LDL-C (mg/dL) } & $78.2 \pm 26.7$ & \multirow{2}{*}{$69.8 \pm 21$} & $71.8 \pm 22.1$ & \multirow[b]{2}{*}{$77.9 \pm 25.2$} \\
\hline & $P=0.003$ & & $P=0.25$ & \\
\hline \multirow{2}{*}{ Estradiol (pg/mL) } & $88.4 \pm 81.9$ & \multirow{2}{*}{$118 \pm 86.9$} & $35.4 \pm 21.7$ & \multirow{2}{*}{$46.9 \pm 41.5$} \\
\hline & $P=0.004$ & & $P=0.1$ & \\
\hline
\end{tabular}

IGF-1: Insulin-like growth factor-1; HOMA-IR: Homeostasis model assessment of insulin resistance; FBS: Fasting blood sugar; TG: Triglycerides; HDL-C: High-density lipoprotein-cholesterol; LDL-C: Low-density lipoprotein-cholesterol

The distribution of the IGF-1 G>A genotypes was in Hardy-Weinberg equilibrium (HWE) in both $\mathrm{AV}$ patients and controls $\left(\chi^{2}=0\right.$ and $\chi^{2}=0.81$, respectively, $P>0.1)$. Also, the distribution of the MTHFR C677T genotypes was in HWE in both AV patients and healthy individuals $\left(\chi^{2}=0.19\right.$ and $\chi^{2}=0.17$, respectively, $\left.P>0.1\right)$.

Table 3 shows the frequency of IGF-1 genotypes and alleles in all patients (i.e., patients with mild-, moderate-, and severe-AV) and controls. As demonstrated in Table 3 , although the frequencies of the IGF-1 GG genotype (51.3\%) and G allele (72\%) were higher in patients than in controls $(45.9 \%$ and $66.2 \%$, respectively), they did not reach to statistical significance. Males' carrier of IGF-1 A allele had a significantly higher level of TG $(115.4 \pm 59.9 \mathrm{mg} / \mathrm{dL})$ compared to those carriers of the IGF-1 G allele (86.8 $\pm 40.9 \mathrm{mg} / \mathrm{dL}, P=0.04)$.

The frequencies of MTHFR C677T genotypes and alleles in all patients (i.e., patients with mild-, moderate, and severe-AV) and controls are presented in Table 4. As indicated in Table 4, the frequency of the MTHFR T allele was $28.1 \%$ in patients and $24.6 \%$ in controls $(P=0.34)$. The comparison of biochemical parameters in females of both groups demonstrated that the presence of the MTHFR T allele was associated with significantly higher levels of FBS $(80.7 \pm 18.5 \mathrm{mg} / \mathrm{dL})$ and LDL-C $(76.4 \pm 24.4 \mathrm{mg} / \mathrm{dL})$, as well as a significantly lower level of estradiol $(87.5 \pm 71 \mathrm{pg} / \mathrm{mL})$ compared to those carriers of the $\mathrm{C}$ allele $(75 \pm 9.9 \mathrm{mg} / \mathrm{dL}, 69.8 \pm 23.5 \mathrm{mg} / \mathrm{dL}$, and 111.4 $\pm 94.7 \mathrm{pg} / \mathrm{mL}$, respectively).

Table 3. Comparison of the IGF-1 genotype and allele frequencies between patients with mild-, moderate-, and severe-AV and controls

\begin{tabular}{|c|c|c|c|c|c|}
\hline \multirow[t]{2}{*}{ Groups } & \multicolumn{3}{|c|}{$\begin{array}{l}\text { IGF-1 genotypes } \\
\text { n (\%) }\end{array}$} & \multicolumn{2}{|c|}{$\begin{array}{c}\text { IGF-1 alleles } \\
\mathrm{n}(\%)\end{array}$} \\
\hline & GG & GA & AA & G & A \\
\hline Mild-AV $(\mathrm{n}=53)$ & $24(45.3)$ & $23(43.4)$ & $6(11.3)$ & $71(67)$ & $35(33)$ \\
\hline Moderate-AV $(n=43)$ & $26(60.5)$ & $14(32.5)$ & $3(7)$ & $66(76.7)$ & $20(23.3)$ \\
\hline Severe-AV $(n=54)$ & $27(50)$ & $24(44.4)$ & $3(5.6)$ & $79(73.1)$ & $29(26.9)$ \\
\hline All patients $(n=150)$ & 77 (51.3) & $61(40.7)$ & $12(8)$ & $215(72)$ & $85(28)$ \\
\hline Controls $(n=148)$ & $68(45.9)$ & $61(41.3)$ & $19(12.8)$ & $196(66.2)$ & $100(33.8)$ \\
\hline
\end{tabular}

AV: Acne vulgaris; IGF-1: Insulin-like growth factor-1

Comparing genotypes between all patients and controls $\chi^{2}=2.1, \mathrm{p}=0.34$

Comparing genotypes between mild-AV patients and controls $\chi^{2}=0.22, \mathrm{p}=0.89$

Comparing genotypes between moderate-AV patients and controls $\chi^{2}=3.07, \mathrm{p}=0.21$

Comparing genotypes between severe-AV patients and controls $\chi^{2}=2.44, p=0.29$

Comparing alleles between all patients and controls $\chi^{2}=2.3, p=0.12$ 
Table 4. Comparison of the frequency of MTHFR genotypes and alleles between patients with mild-, moderate-, and severe-AV and controls

\begin{tabular}{cccccc}
\hline Groups & \multicolumn{3}{c}{$\begin{array}{c}\text { MTHFR genotypes } \\
\mathrm{n}(\%)\end{array}$} & \multicolumn{2}{c}{$\begin{array}{c}\text { MTHFR alleles } \\
(\%)\end{array}$} \\
\hline Mild-AV $(\mathrm{n}=56)$ & $31(55.4)$ & $21(37.5)$ & $4(7.1)$ & $83(74.1)$ & $29(25.9)$ \\
\hline Moderate-AV $(\mathrm{n}=43)$ & $21(48.8)$ & $18(41.9)$ & $4(9.3)$ & $60(69.8)$ & $26(30.2)$ \\
\hline Severe-AV $(\mathrm{n}=54)$ & $26(48.1)$ & $25(46.3)$ & $3(5.6)$ & $77(71.3)$ & $31(28.7)$ \\
\hline All patients $(\mathrm{n}=153)$ & $78(51)$ & $64(41.8)$ & $11(7.2)$ & $220(71.9)$ & $86(28.1)$ \\
\hline Controls $(\mathrm{n}=134)$ & $77(57.5)$ & $48(35.8)$ & $9(6.7)$ & $202(75.4)$ & $66(24.6)$ \\
\hline
\end{tabular}

MTHFR: 5,10-methylenetetrahydrofolate reductase; AV: Acne vulgaris

Comparing genotypes between all patients and controls $\chi^{2}=1.24, \mathrm{p}=0.53$

Comparing genotypes between mild-AV patients and controls $\chi^{2}=0.03, \mathrm{p}=0.98$

Comparing genotypes between moderate-AV patients and controls $\chi^{2}=0.84, \mathrm{p}=0.65$

Comparing genotypes between severe-AV patients and controls $\chi^{2}=1.262, \mathrm{p}=0.44$

Comparing alleles between all patients and controls $\chi^{2}=0.88, p=0.34$

\section{Discussion}

Various factors including genetic background, nutrition and environment affect the incidence of AV. Previously we examined the role of some genetic variants and also hormonal factors and lipid profile in susceptibility to AV $(2,7)$.

In the present study, the higher serum levels of insulin, HOMA, and HDL-C were detected in all patients with AV compared to controls. The genderspecific differences were observed in the biochemical characteristics of patients and controls. In females, increased insulin, HOMA, total cholesterol, and LDL$\mathrm{C}$ levels, as well as decreased estradiol levels, were associated with the presence of AV. Changes in lipid profile can be considered in the treatment of female patients with moderate to severe AV (11). On the other hand, estradiol, the major female estrogenic hormone, may prevent the production of androgen and thus decrease the growth of the sebaceous gland and thereby decrease sebaceous gland growth (12). Therefore, the increased level of estradiol reduces the risk of $\mathrm{AV}$ and its severity. In males, just increased cholesterol was associated with AV. In one available study, among males with AV, it was shown that HDL-C and insulin could be involved in AV development (13).

In our study, the serum level of insulin was significantly higher in patients than in healthy individuals. The comparison between different types of $\mathrm{AV}$ showed the absence of an association between the insulin level and the severity of AV. However, insulin and HOMA were associated with the risk of AV in females but not in males. Some endocrine diseases can be associated with the AV risk. In females with $\mathrm{AV}$, similar to patients with polycystic ovary syndrome (PCOS), insulin resistance is known to be a risk factor, which changes the level of androgens (13). In patients with PCOS, insulin resistance and hyperinsulinemia play an important role in the molecular mechanisms involved in ovarian androgenic hyper-secretion typical of PCOS (14)

On the other hand, insulin and IGF-1 cause hormonal changes in adrenal and gonadal androgen synthesis (15). Changes in androgens and hormonal mediators are effective in the production of sebum and consequently AV susceptibility (16). Several studies presented different results about the relationship between the AV risk and the IGF-1 level. Behrangi et al. found that the mean serum level of IGF-1 was significantly associated with AV (17). However, there was no association between the severity of AV and the IGF-1 level. In another study, IGF-1 had a stronger effect on acne in women compared to men, but androgens played a greater role in acne among men (18). According to both studies, gender difference can influence the IGF-1 level in patients with AV. In our study, although the serum level of IGF-1 was higher in patients compared to controls, its difference was near to significant. Thus, the level of IGF-1 may almost be a possible pathogenic factor in $\mathrm{AV}$.

In the human body, the IGF-1 gene encodes a protein that has a similar insulin function and structure. It is a significant contributor to cellular growth and development, protein translation, differentiation, metabolism, apoptosis, etc. Also, this gene may influence the androgen secretion and thus be effective in the pathogenesis of AV (19). It seems that among the Turkish population IGF-1 (cytosine adenosine, CA) 19 polymorphism is associated with the predisposition to AV (20). Also, Rahaman et al. found an association between IGF-1 (cytosine adenosine) 19 polymorphism with AV and its severity (21). In the current study, we investigated another polymorphism of IGF-1 G>A and found the absence of an association between this polymorphism and the risk of AV. 
The MTHFR polymorphism was also evaluated by considering its key role in the pathway of folate metabolism and DNA, RNA, and protein methylation. A frequency of $27.85 \%$ for the MTHFR T allele has been reported among healthy individuals from Western Iran (22). In the present study, the MTHFR C677T polymorphism had no relationship with the AV susceptibility. However, among women, our study detected that in the presence of MTHFR T allele the levels of FBS and LDL-C were higher and the level of estradiol was lower compared to the wild allele of MTHFR (C). The MTHFR C677T polymorphism has been associated with hyperlipidemia in Indian women with PCOS, and carriers of the MTHFR CT genotype had a significantly higher level of TG and cholesterol compared to carriers of the MTHFR CC genotype (23). However, among Korean women with PCOS, no relationship was reported between the MTHFR C677T polymorphism and the risk of PCOS (24).

\section{Conclusion}

Our study demonstrated the lack of association between the IGF-1 and the MTHFR C677T polymorphism with the risk of AV. However, insulin, IGF-1, and HOMA-IR levels increased in AV patients compared to healthy individuals, which indicated the influence of insulin and insulin resistance in the risk of AV and its severity. We found that among women the MTHFR C677T polymorphism was associated with higher FBS and LDL-C levels, as well as lower levels of estradiol.

\section{Acknowledgements}

This work was performed in partial fulfillment of the requirements for MSc degree of Sakineh Zinati and was financially supported by a grant from Vice Chancellor for Research of Kermanshah University of Medical Sciences, Kermanshah, Iran.

\section{Conflict of Interest}

The authors declared that there is no conflict of interest regarding the publication of this article.

\section{References}

1. Morze J, Przybylowicz KE, Danielewicz A, ObaraGolebiowska M. Diet in Acne Vulgaris: Open or Solved Problem?. Iran J Public Health 2017; 46: 428-430.

2. Saeidi S, Chamaie-Nejad F, Ebrahimi A, et al. PPAR $\gamma$ Pro12Ala and C161T polymorphisms in patients with acne vulgaris: Contribution to lipid and lipoprotein profile. Adv. Med. Sci 2018; 63:147-151. [DOI:10.1016/j.advms.2017.09.003] [PMID]

3. Harper ME, A. Ullrich, Saunders GF. Localization of the human insulin gene to the distal end of the short arm of chromosome 11. Proc. Natl. Acad. Sci 1981; 78: 44584460. [DOI:10.1073/pnas.78.7.4458] [PMID] [PMCID]
4. Melnik BC, Schmitz G. Role of insulin, insulin-like growth factor-1, hyperglycaemic food and milk consumption in the pathogenesis of acne vulgaris. Exp. Dermatol 2009; 18: 833-841. [DOI:10.1111/j.1600-0625.2009.00924.x] [PMID]

5. Rahimi Z, Malek-Khosravi S, Rahimi Z, Jalilvand F, Parsian A. MTHFR C677T and eNOS G894T variants in preeclamptic women: Contribution to lipid peroxidation and oxidative stress. Clin. Biochem 2013; 46: 143-147. [DOI:10.1016/j.clinbiochem.2012.10.020] [PMID]

6. JafariY, Rahimi Z, Vaisi-Raygani A, Rezaei M. Interaction of eNOS polymorphism with MTHFR variants increase the risk of diabetic nephropathy and its progression in type 2 diabetes mellitus patients. Mol. Cell. Biochem 2011; 353: 23-34. [DOI:10.1007/s11010-011-0770-0] [PMID]

7. Chamaie-Nejad F, Saeidi S, Najafi F, et al. Association of the CYP17 MSP AI (T-34C) and CYP19 codon 39 (Trp/Arg) polymorphisms with susceptibility to acne vulgaris. Clin. Exp. Dermatol 2018; 43: 183-186. [DOI:10.1111/ced.13321] [PMID]

8. Rahimi Z, Felehgari V, Rahimi M, et al. The frequency of factor $\mathrm{V}$ Leiden mutation, ACE gene polymorphism, serum ACE activity and response to ACE inhibitor and angiotensin II receptor antagonist drugs in Iranians type II diabetic patients with microalbuminuria. Mol Biol Rep. 2011; 38: 2117-23. [DOI:10.1007/s11033-010-0338-1] [PMID]

9. Karimi K, Mahmoudi T, Karimi N, et al. Is there an association between variants in candidate insulin pathway genes IGF-I, IGFBP-3, INSR, and IRS2 and risk of colorectal cancer in the Iranian population? Asian. Pac. J. Cancer Prev 2013; 14: 5011-6. [DOI:10.7314/APJCP.2013.14.9.5011] [PMID]

10. Rahimi M, Hasanvand A, Rahimi Z, et al. Synergistic effects of the MTHFR C677T and A1298C polymorphisms on the increased risk of micro- and macroalbuminuria and progression of diabetic nephropathy among Iranians with type 2 diabetes mellitus. Clin. Biochem 2010; 43: 1333-1339. [DOI:10.1016/j.clinbiochem.2010.08.019] [PMID]

11. Bakry OA, El Shazly RMA, El Farargy SM, Kotb D. Role of hormones and blood lipids in the pathogenesis of acne vulgaris in non-obese, non-hirsute females. Indian Dermatol. Online J 2014; 5: S9. [DOI:10.4103/22295178.144506] [PMID] [PMCID]

12. Thiboutot D. Hormones and acne: pathophysiology, clinical evaluation, and therapies. Semin. Cutan. Med. Surg 2001; 20:144-153. [DOI:10.1053/sder.2001.28208] [PMID]

13. Del Prete M, Mauriello MC, Faggiano A, et al. Insulin resistance and acne: a new risk factor for men?. Endocrine 2012; 42: 555-560. [DOI:10.1007/s12020-012-9647-6] [PMID]

14. Rojas J, Chávez M, Olivar L, Rojas M, Morillo J, Mejías $\mathrm{J}$, et al. Polycystic ovary syndrome, insulin resistance, and obesity: navigating the pathophysiologic labyrinth. International journal of reproductive medicine. 2014;2014. [DOI:10.1155/2014/719050] [PMID] [PMCID]

15. Kumari R, Thappa DM. Role of insulin resistance and diet in acne. Indian J. Dermatol. Venereol. Leprol 2013; 79: 291. [DOI:10.4103/0378-6323.110753] [PMID] 
16. Pappas A. The relationship of diet and acne: a review. Dermatoendocrinol 2009; 1: 262-267. [DOI:10.4161/derm.1.5.10192] [PMID] [PMCID]

17. Behrangi E, Rasi A, Navid P, Dalvand B, Azizian Z. Serum Level of Insulin-Like Growth Factor 1 in Patients with Acne Vulgaris Versus Healthy Subjects. J. Stem Cell 2018; 5 (3): e79784. [DOI:10.5812/jssc.79784]

18. Cappel M, Mauger D, Thiboutot D. Correlation between serum levels of insulin-like growth factor 1, dehydroepiandrosterone sulfate, and dihydrotestosterone and acne lesion counts in adult women. Arch. Dermatol 2005; 141: 333-338 [DOI:10.1001/archderm.141.3.333] [PMID]

19. Clatici VG, Georgescu O, Draganita AMV, Tatu AL, Fica S. Milk and Insulin Growth Factor 1 (IGF1)-Implication in Acne and General Health. Rom. Biotechnol. Lett 2015; 20: 10013.

20. Tasli L, Turgut S, Kacar N, Ayada C, Coban M, Akcilar R, Ergin S. Insulinlike growth factorI gene polymorphism in acne vulgaris. J.Eur. Acad. Dermatol. Venereol
2013;27(2):254-7. 3083.2011.04299.x] [PMID]

[DOI:10.1111/j.1468

21. Rahaman SMA, De D, Handa S, et al. Association of insulin-like growth factor (IGF)-1 gene polymorphisms with plasma levels of IGF-1 and acne severity. J. Am Acad. Dermatol 2016; 75: 768-773. [DOI:10.1016/j.jaad.2016.05.019] [PMID]

22. Rahimi Z, Ghaderi M, Nagel RL, Muniz A. Prevalence of thrombotic risk factors among $\beta$-thalassemia patients from Western Iran. J. Thromb. Thrombolysis 2008; 26: 229233. [DOI:10.1007/s11239-007-0163-0] [PMID]

23. Jain M, Pandey P, Tiwary NK, Jain S. MTHFR C677T polymorphism is associated with hyperlipidemia in women with polycystic ovary syndrome. J. Hum. Reprod. Sci 2012; 5: 52-56. [DOI:10.4103/0974-1208.97802] [PMID] [PMCID]

24. Choi SW, Gu BH, Ramakrishna S, Park JM, Baek KH. Association between a single nucleotide polymorphism in MTHFR gene and polycystic ovary syndrome. Eur. J. Obstet. Gynecol. Reprod. Biol 2009; 145: 85-88. [DOI:10.1016/i.ejogrb.2009.04.013] [PMID]

\section{How to Cite This Article}

Zinati-Saeed, S., Shakiba, E., Rahimi, Z., Akbari, M., Najafi, F., Bahrehmand, F., Vaisi-Raygani, A., Rahimi, Z., Ebrahimi, A., Rahimi, M. The Insulin-like Growth Factor-1 (G>A) and 5,10-methylenetetrahydrofolate Reductase (C677T) Gene Variants and the Serum Levels of Insulin-like Growth Factor-1, Insulin, and Homeostasis Model Assessment in Patients with Acne Vulgaris. Iranian Journal of Pathology, 2019; 15(1): 23-29. doi: 10.30699/ijp.2019.105695.2098 